\title{
Time-Resolved Photoluminescence Spectroscopy Evaluation of CdTe and CdTe/CdS Quantum Dots
}

\author{
Zhimin Yuan, Ping Yang, and Yongqiang Cao \\ School of Material Science and Engineering, University of Jinan, Jinan 250022, China \\ Correspondence should be addressed to Ping Yang, mse_yangp@ujn.edu.cn
}

Received 2 August 2012; Accepted 7 September 2012

Academic Editors: U. Pal and C. Tormena

Copyright () 2012 Zhimin Yuan et al. This is an open access article distributed under the Creative Commons Attribution License, which permits unrestricted use, distribution, and reproduction in any medium, provided the original work is properly cited.

CdTe and CdTe/CdS quantum dots (QDs) were prepared in aqueous solutions using thioglycolic acid as a stabilizing agent. The photoluminescence (PL) wavelength of the QDs depended strongly on the size of CdTe cores and the thickness of CdS shells. Being kept at room temperature for 130 days, the PL wavelength of CdTe and CdTe/CdS QDs was red-shifted. However the red-shifted degree of CdTe QDs is larger than that of CdTe/CdS QDs. The size of CdTe QDs and the thickness of CdS play important roles for the red-shift of PL spectra of CdTe/CdS QDs. In contrast, the full width at half maximum of PL spectra of CdTe and CdTe/CdS QDs almost remained unchanged. This is ascribed to the effects of $\mathrm{Cd}^{2+}$ and TGA in solutions on the growth of CdTe and CdTe/CdS QDs. This associated with the variation of surface state of the QDs during store. The results demonstrate that CdTe/CdS core/shell QDs have high stability compared with CdTe QDs due to a CdS shell.

\section{Introduction}

Quantum dots (QDs) have attracted considerable interest in the past two decades because of their excellent properties such as narrower and symmetric emission spectra, broad absorption spectra, and better photostability than traditional fluorescent labels [1-5]. An organic synthetic approach in trioctylphosphine or trioctylphosphine oxide at high temperature has been well developed to prepare highly luminescent II-VI QDs [6]. However, the QDs are insoluble in an aqueous solution and not compatible for biological applications. Compared with organic strategies, an alternative approach to produce water soluble QDs is to directly synthesize QDs in an aqueous solvent. Such approach is less expensive, highly reproducible, less toxic, and more biocompatible [7]. It is easy to control QD size in an aqueous approach because of a low nucleation and growth rate.

Early aqueous CdTe QDs with a low stability were prepared in the presence of thioglycolic acid (TGA) as the capping agent by using a reaction between $\mathrm{Cd}^{2+}$ and NaHTe solution [8]. These QDs were easily subjected to the photooxidation and photobleaching when used to labeling in living cells, resulting in significant PL quenching [9]. To improve the photostability, epitaxial growth of an inorganic shell on the surface of CdTe QDs in aqueous solution was explored in a few research groups, similar to growing a shell of $\mathrm{ZnS}, \mathrm{CdS}$, or $\mathrm{ZnSe}$ on CdSe cores in organic solutions [6, 10-13]. It has been illustrated that epitaxial growth of an inorganic shell with a broader band gap on the surface of luminescent QDs is an ideal approach to improve the properties of QDs through the elimination of both the anionic and the cationic surface dangling bonds on the QDs, which leads to an enhancement of stability [14]. CdS is in principle a good candidate as the shell material for CdTe cores not only because its band gap $(2.5 \mathrm{eV})$ is wider than that of CdTe $(1.5 \mathrm{eV})$ but also because its lattice parameter mismatch $(3.6 \%)$ to CdTe is relatively small compared with $\mathrm{ZnSe}(12.5 \%)$ and $\mathrm{ZnS}$ $(16.5 \%)$ [10, 15, 16]. Wang and coworkers reported on the preparation of 1-thioglycerol-capped CdTe/CdS QDs by the ultrasonic irradiation [17]. Peng's group prepared TGAcapped CdTe/CdS QDs with enhanced photostability [18]. Overcoating CdS layer outside of the CdTe core has become an important stratagem to enhance the photostability of QDs. 
In this work, TGA-capped CdTe and CdTe/CdS QDs have been synthesized in an aqueous solution. The structural and optical properties of CdTe and CdTe/CdS QDs were characterized and studied by transmission electron microscopy (TEM), X-ray diffraction (XRD), and optical spectroscopy. The thickness of the CdS shell was accurately controlled by changing the stoichiometry of the injection solutions and the growth condition. In particular we have performed the experiments on the thickness effect of CdS shell on the PL dynamics of CdTe/CdS QDs. Finally the storage experiment was carried out to examine the high photostability of as-prepared CdTe/CdS QDs. Systematic investigations demonstrated that the $\mathrm{CdTe} / \mathrm{CdS}$ core/shell QDs revealed a prolonged average PL lifetime and good PL stability properties than CdTe core QDs. We expect that the unique optical properties of the CdTe/CdS core/shell QDs reported herein will advance understanding of CdTe QDs and will suggest new applications of QDs.

\section{Material and Methods}

2.1. Materials. TGA (99\%), tellurium powder (Te, 99\%), $\mathrm{CdCl}_{2} \cdot 2.5 \mathrm{H}_{2} \mathrm{O}(98 \%)$, and sodium borohydride $\left(\mathrm{NaBH}_{4}\right)$ (96\%) were purchased from Shanghai Chemical Reagents Company. Sodium sulfide $\left(\mathrm{Na}_{2} \mathrm{~S} \cdot 9 \mathrm{H}_{2} \mathrm{O}\right)$ was supplied from Tianjin Damao Chemical Reagents Institute. The pure water was obtained from a Milli-Q synthesis system. All chemicals mentioned in the current investigations were used as received.

2.2. Synthesis of Water-Soluble TGA-Capped CdTe QDs. Aqueous TGA-capped CdTe QDs were prepared by a mild method according to the procedure reported in literature [19]. Firstly synthesize sodium hydrogen telluride (NaHTe) solution by reacting $\mathrm{NaBH}_{4}$ with $\mathrm{Te}$ ( $5 / 1$ molar ratio). Briefly, $1 \mathrm{mmol}$ of $\mathrm{NaBH}_{4}$ and $0.2 \mathrm{mmol}$ of Te were added to a small two-neck flask and protected by $\mathrm{N}_{2}$ for $30 \mathrm{~min}$. Secondly, $0.4 \mathrm{mmol} \mathrm{CdCl}_{2} \cdot 2.5 \mathrm{H}_{2} \mathrm{O}$ was dissolved in $25 \mathrm{~mL}$ water and $0.6 \mathrm{mmol}$ TGA was added followed by adjusting $\mathrm{pH}$ to 11 with $1 \mathrm{M} \mathrm{NaOH}$ solution under vigorous stirring. When the mixture had been degassed by high-purity $\mathrm{N}_{2}$ for $30 \mathrm{~min}$, a certain amount of oxygen-free NaHTe solution was injected. Finally the complex solution with a faint yellow color was refluxed at $100^{\circ} \mathrm{C}$ for a certain time. Different-sized CdTe QDs were prepared by varying the reflux time.

2.3. Synthesis of TGA-Stabilized CdTe/CdS QDs. Synthesis of $\mathrm{CdTe} / \mathrm{CdS}$ core/shell QDs is described as follows. $10 \mathrm{~mL}$ asprepared CdTe colloidal solution was precipitated using 2propanol mixed with ethanol and then redispersed in $20 \mathrm{~mL}$ of $\mathrm{H}_{2} \mathrm{O}$ including $0.2 \mathrm{mmol}$ of $\mathrm{CdCl}_{2} \cdot 2.5 \mathrm{H}_{2} \mathrm{O}$ and $0.3 \mathrm{mmol}$ of TGA to get solution $\mathrm{A}(\mathrm{PH}=11$, adjust by $1 \mathrm{M} \mathrm{NaOH})$. $0.4 \mathrm{mmol}$ of $\mathrm{Na}_{2} \mathrm{~S} \cdot 9 \mathrm{H}_{2} \mathrm{O}$ was dissolved in $40 \mathrm{~mL}$ of pure water to get solution $\mathrm{B}$. Both solutions $\mathrm{A}$ and $\mathrm{B}$ were bubbled by $\mathrm{N}_{2}$ for $1 \mathrm{~h}$. When the solution $\mathrm{A}$ was heated to $100^{\circ} \mathrm{C}$, solution $B$ was added using an injection rate of $1 \mathrm{~mL} / \mathrm{min}$ with stirring. For controlling the thickness of CdS shell, $1.5 \mathrm{~mL}$ and $3.5 \mathrm{~mL}$ of solution $\mathrm{B}$ were added to solution $\mathrm{A}$ to get different size CdTe/CdS core/shell QDs.
2.4. Characterization. All optical measurements were performed at room temperature under ambient conditions. The absorption spectra were recorded with a HITACHI U-4100 UV-vis-NIR Spectrophotometer. Integrated PL measurements were performed using a HITACHI F-4600 Fluorescence Spectrophotometer. The sizes of QDs were determined by a TEM (Hitachi H9000NA). Samples were precipitated by 2-propanol and dried in a vacuum oven for X-ray diffractometer (XRD) characterization with a Bruker D8 diffractometer. The PL lifetimes were measured using a time-correlated single-photon-counting spectrofluorometer system (Fluorocube-01, JY-IBH, Horiba). The recordshifted decay curves were fitted with a biexponential function by a least squares fitting method.

\section{Results and Discussion}

3.1. PL Properties and Crystal Structure of CdTe/CdS QDs. Figure 1 shows the PL and absorption spectra of TGA-capped CdTe cores and CdTe/CdS core/shell QDs list in Table 1 (as-prepared). By comparison, Figure 2 shows the PL and absorption spectra of TGA-capped CdTe cores and CdTe/CdS core/shell QDs list in Table 1 (130 days). Table 1 summarizes the characteristics of the optical properties of the CdTe cores and $\mathrm{CdTe} / \mathrm{CdS}$ core/shell QDs. In Figures 1 and 2, the PL and absorption spectra of TGA-capped CdTe/CdS core/shell QDs have a significant red shift and the full width at half maximum (fwhm) decreased compared with CdTe cores. For example, the emission peaks $\left(\lambda_{\mathrm{em}}\right)$ of $\mathrm{CdTe} / \mathrm{CdS}$ core/shell QDs shift from $541 \mathrm{~nm}$ for CdTe core QDs (sample A0) to $578 \mathrm{~nm}$ for CdTe/CdS core/shell QDs (sample A3), when the sample is as-prepared. The $\lambda_{\mathrm{em}}$ of CdTe/CdS core/shell QDs shift from $556 \mathrm{~nm}$ for CdTe core QDs (sample A0) to $585 \mathrm{~nm}$ for $\mathrm{CdTe} / \mathrm{CdS}$ core/shell QDs (sample A3), when the storage time is 130 days. In addition, another important requirement for QDs is their fwhm. Increased fwhm indicates a broader particle size distribution. It is noteworthy that our $\mathrm{CdTe} / \mathrm{CdS}$ emitting QDs possess an extremely narrow fwhm of the PL band ranging from 39 to $46 \mathrm{~nm}$ (as-prepared) and from 38 to $47 \mathrm{~nm}$ (130 days), which can be used directly in a chemical or biological system without any extra purification.

From the data list in Table 1, we know that the $\lambda_{\mathrm{em}}$ of sample A0 shifted from 541 (as-prepared) to $556 \mathrm{~nm}$ (130 days) and simultaneously the fwhm of sample A0 decreased from $40 \mathrm{~nm}$ to $38 \mathrm{~nm}$. For the phenomena, the main reason is that in the first stage of CdTe nucleating and growing, the surface of CdTe QDs has many defects. Due to Ostwald ripening mechanism little particles dissolved and big particles grew; consequently the $\lambda_{\mathrm{em}}$ of sample A0 have a red shift and the fwhm of sample A0 has a decrease. The $\lambda_{\mathrm{em}}$ of sample A1 shift from 561 (as-prepared) to $572 \mathrm{~nm}$ (130 days), while the fwhm of sample A1 increases from $39 \mathrm{~nm}$ to $40 \mathrm{~nm}$. Compared with sample A0, sample A1 has a CdS shell and better stability, which is shown in Figures 1 and 2. The red shift of $\lambda_{\mathrm{em}}$ from as-prepared to 130 days for samples A0, $\mathrm{A} 1$, and $\mathrm{A} 2$ were 15,11 , and $7 \mathrm{~nm}$, respectively. The shorter red shift of CdTe/CdS core/shell QDs demonstrates better stability compared with core CdTe QDs. Groups B, C, and 

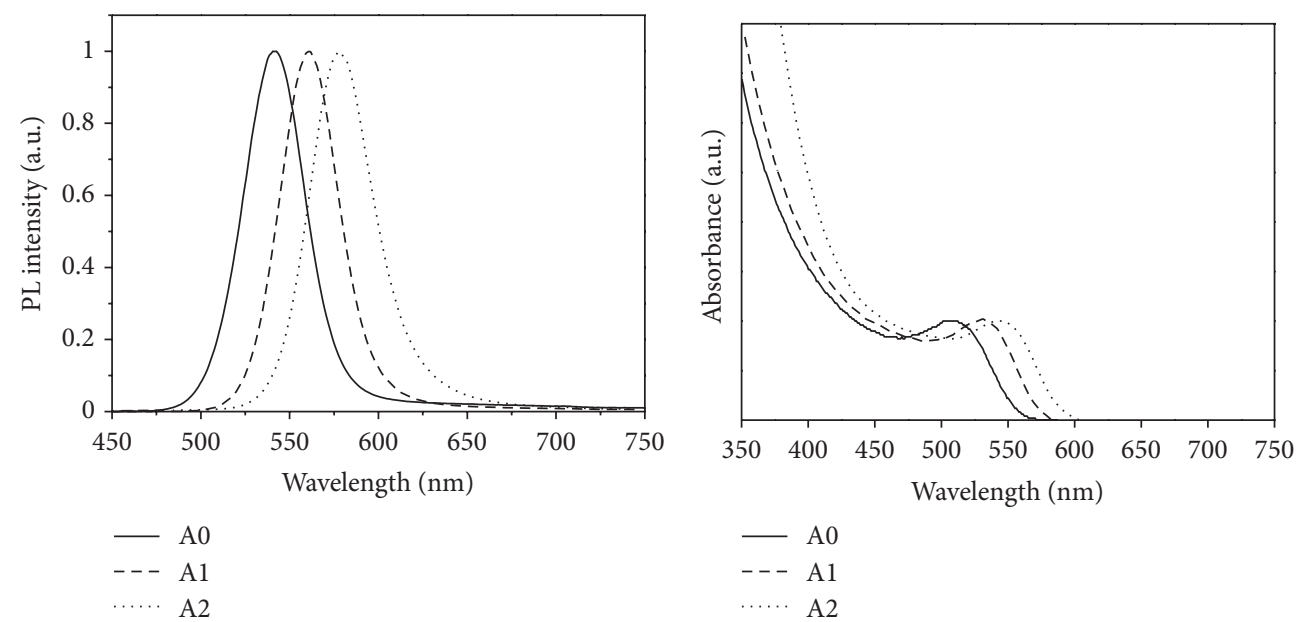

(a)

(b)
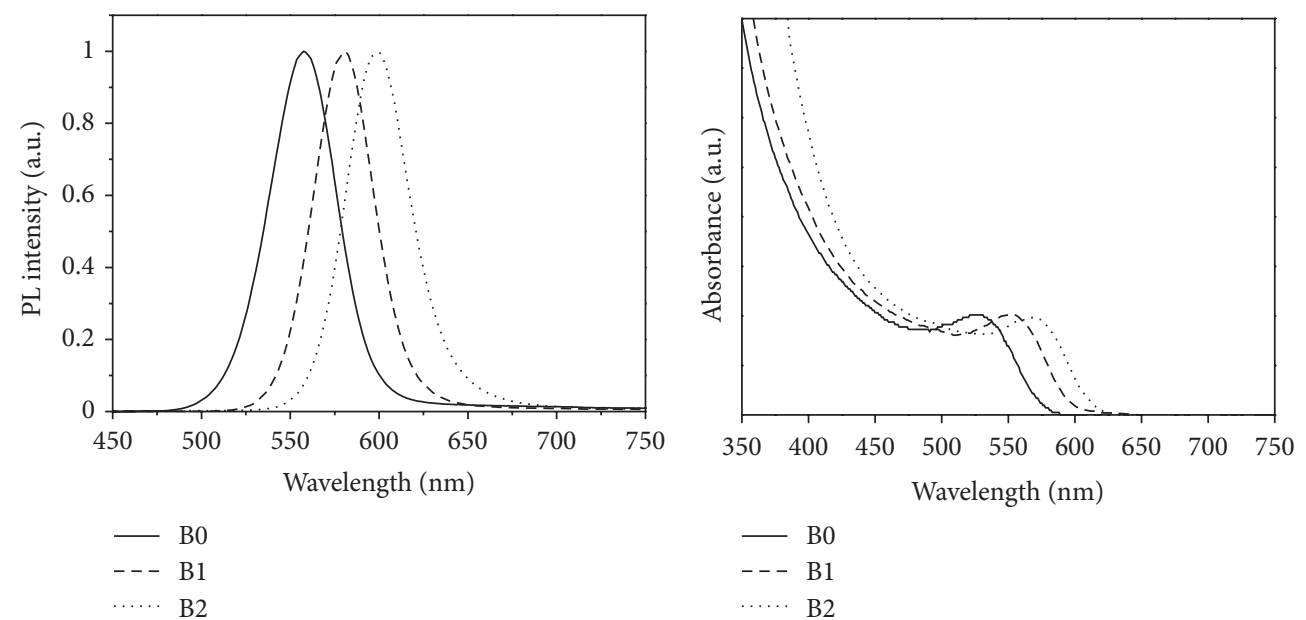

…. B2

(c)

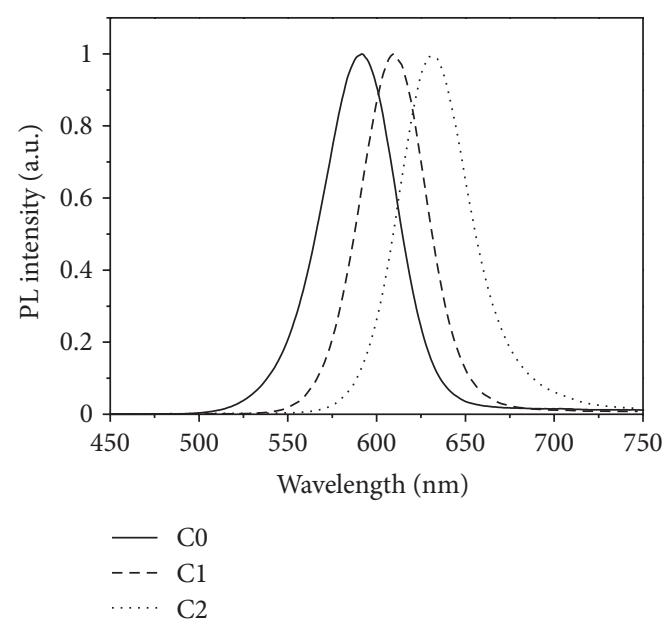

(e)

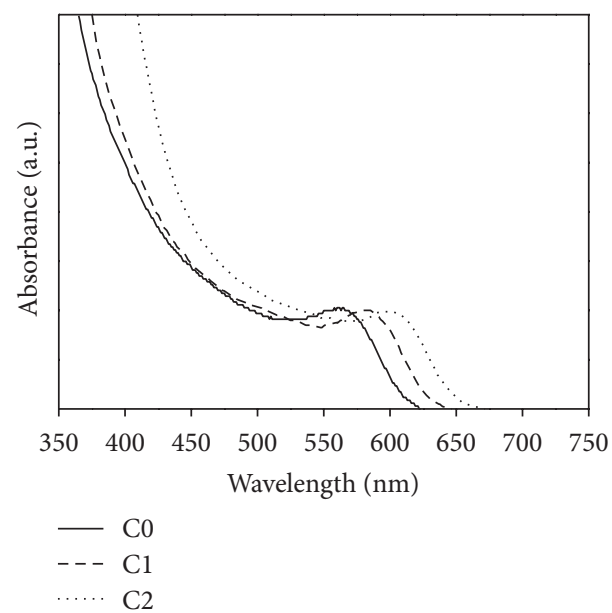

(f)

Figure 1: PL and absorption spectra of TGA-capped CdTe cores and CdTe/CdS core/shell QDs as shown in Table 1 (as-prepared). (a) PL spectra of samples A0, A1, and A2; (b) absorption spectra of samples A0, A1, and A2; (c) PL spectra of samples B0, B1, and B2; (d) absorption spectra of samples B0, B1, and B2; (e) PL spectra of samples C0, C1, and C2; (f) absorption spectra of samples C0, C1, and C2. 

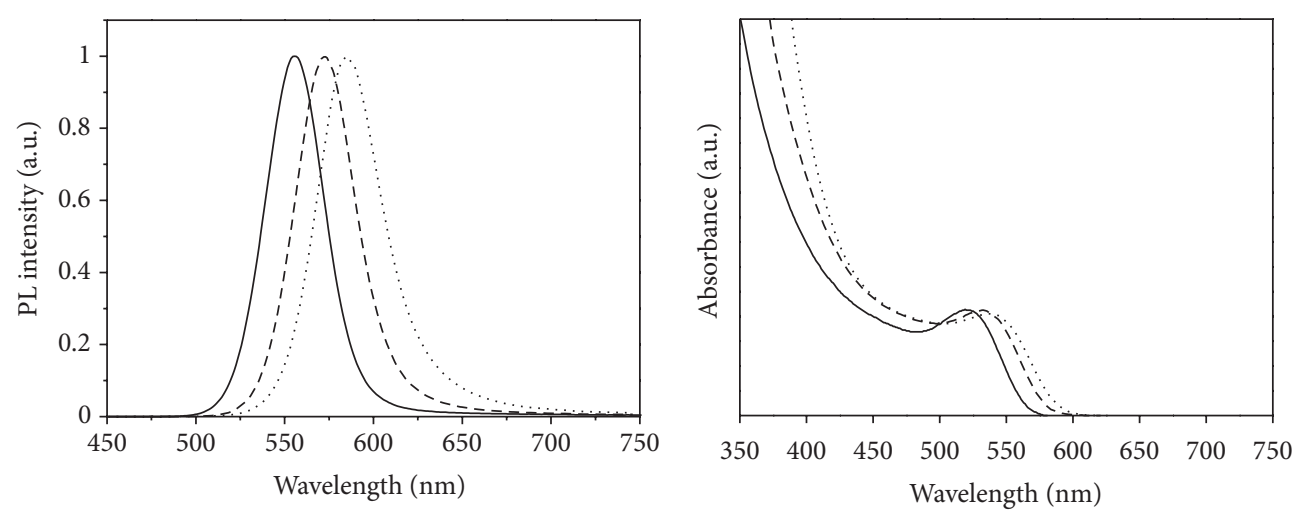

$$
\begin{array}{ll}
- & \mathrm{A} 0 \\
--- & \mathrm{A} 1 \\
\cdots \cdots & \mathrm{A} 2
\end{array}
$$

(a)

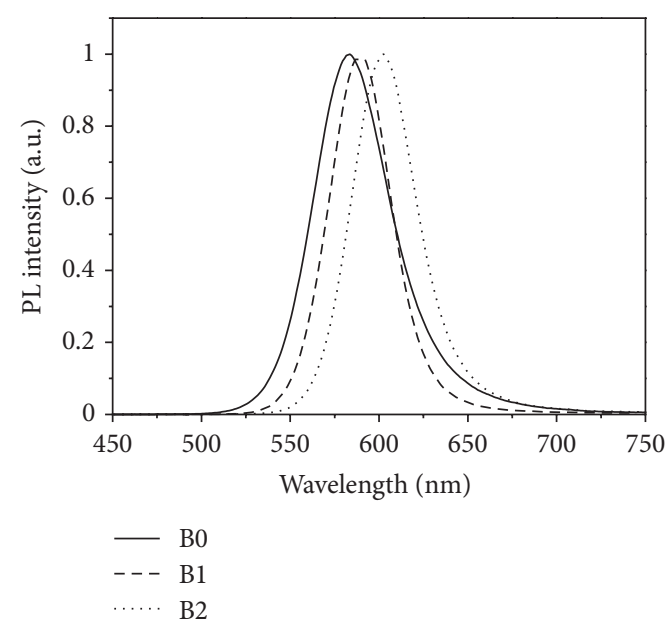

(c)

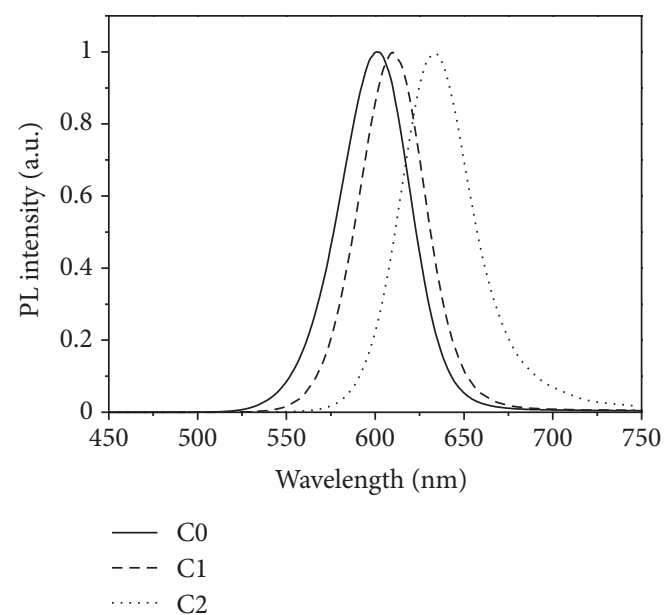

(e)

$$
\begin{array}{ll}
- & \mathrm{A} 0 \\
--- & \mathrm{A} 1 \\
\cdots \cdots & \mathrm{A} 2
\end{array}
$$

(b)

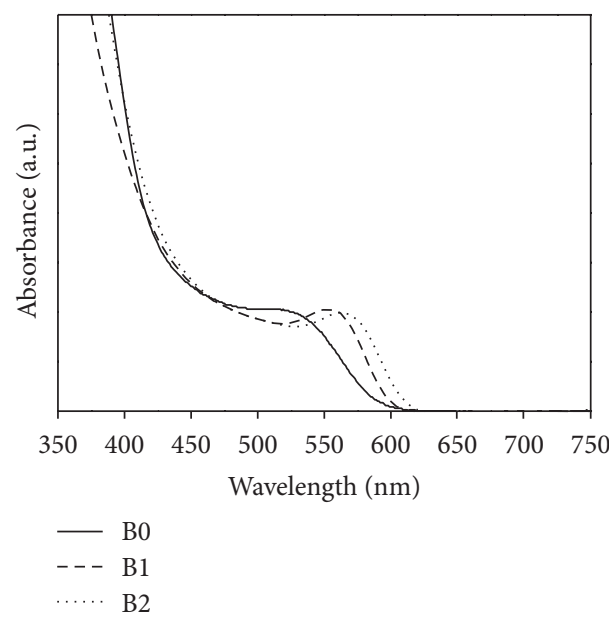

(d)

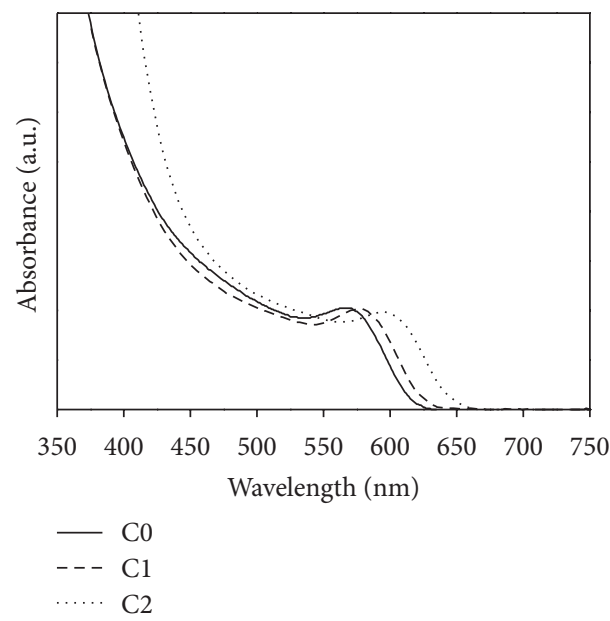

(f)

Figure 2: PL and absorption spectra of TGA-capped CdTe cores and CdTe/CdS core/shell QDs as shown in Table 1 (130 days). (a) PL spectra of samples A0, A1, and A2; (b) absorption spectra of samples A0, A1, and A2; (c) PL spectra of samples B0, B1, and B2; (d) absorption spectra of samples B0, B1, and B2; (e) PL spectra of samples C0, C1, and C2; (f) absorption spectra of samples C0, C1, and C2. 
TABLE 1: PL properties of TGA-capped CdTe and CdTe/CdS core/shell QDs.

\begin{tabular}{|c|c|c|c|c|c|c|}
\hline Sample & QD kinds & $\lambda_{\mathrm{em}} / \mathrm{nm}$ as-prepared & fwhm/nm as-prepared & $\lambda_{\mathrm{em}} / \mathrm{nm} 130$ days & fwhm/nm 130 days & Red shift/nm \\
\hline A0 & $\mathrm{CdTe}$ & 541 & 40 & 556 & 38 & 15 \\
\hline A1 & $\mathrm{CdTe} / \mathrm{CdS}$ & 561 & 39 & 572 & 40 & 11 \\
\hline A2 & $\mathrm{CdTe} / \mathrm{CdS}$ & 578 & 43 & 585 & 43 & 7 \\
\hline B0 & $\mathrm{CdTe}$ & 557 & 44 & 582 & 50 & 25 \\
\hline B1 & $\mathrm{CdTe} / \mathrm{CdS}$ & 580 & 40 & 589 & 41 & 9 \\
\hline B2 & $\mathrm{CdTe} / \mathrm{CdS}$ & 598 & 41 & 602 & 43 & 4 \\
\hline $\mathrm{C} 0$ & CdTe & 591 & 48 & 601 & 47 & 10 \\
\hline $\mathrm{C} 1$ & $\mathrm{CdTe} / \mathrm{CdS}$ & 610 & 44 & 610 & 43 & 0 \\
\hline $\mathrm{C} 2$ & $\mathrm{CdTe} / \mathrm{CdS}$ & 631 & 46 & 633 & 47 & 2 \\
\hline
\end{tabular}

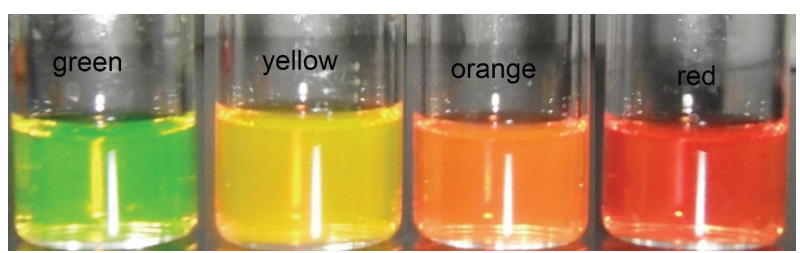

Figure 3: The photographs are aqueous solution of $\mathrm{CdTe}$ and $\mathrm{CdTe} / \mathrm{CdS}$ core/shell QDs. They were taken under normal room light without using additional excitation light source. The green to red colors are from the fluorescence of $\mathrm{CdTe}$ and $\mathrm{CdTe} / \mathrm{CdS}$ core/shell QDs.

$\mathrm{D}$ have analogical phenomena with group A. Sample B0 may be a special case; the red shift of $\lambda_{\text {em }}$ from as-prepared to 130 days is $25 \mathrm{~nm}$. The size of sample B0 has a great increase due to the slight aggregation and instability of CdTe core QDs. From the datas list in Table 1, a conclusion can be drawn that longer $\lambda_{\mathrm{em}}$ of $\mathrm{CdTe} / \mathrm{CdS}$ core/shell QDs have better stability than shorter ones. For example, sample $\mathrm{C} 1$ has $0 \mathrm{~nm}$ red shift and sample $\mathrm{C} 2$ has $2 \mathrm{~nm}$ red shift, while sample B1 has $9 \mathrm{~nm}$ red shift and sample B2 has $4 \mathrm{~nm}$ red shift.

Figure 3 shows the photographs of aqueous solution $\mathrm{CdTe}$ and CdTe/CdS core/shell QDs. They were taken under normal room light without using additional excitation light source. The luminescent color varied from green to red. In Figure 3 the sample shows well photoluminescent properties. To confirm the CdS shell formation on the CdTe cores, Figure 4 shows the XRD patterns of CdTe cores and CdTe/CdS core/shell QD. The CdTe cores (sample C0) exhibited a diffraction pattern quite close to that of bulk cubic CdTe. The dominant structure of the core is the CdTe crystal phase. In contrast, the diffraction pattern of the $\mathrm{CdTe} / \mathrm{CdS}$ QDs (sample C2) slightly moves toward higher angles with peak widths and shapes being nearly unchanged, which is close to the diffraction peak of cubic CdS. Our results are similar to the changes observed in the diffraction pattern of CdTe cores when a CdS shell grows on them $[17,20]$.

To characterize the quality of the samples, typical TEM images of CdTe cores and corresponding CdTe/CdS core/shell QDs with different CdS shell thickness are shown in Figure 5. Figure 5 shows the TEM images of CdTe core

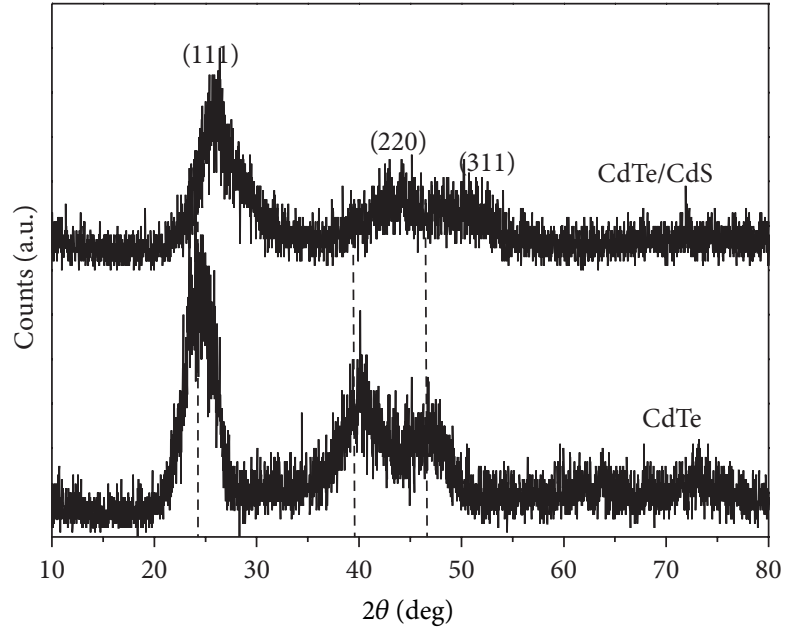

FIGURE 4: XRD patterns of TGA-capped CdTe QDs and CdTe/CdS core/shell QDs shown in Table 1. CdTe: sample C0; CdTe/CdS: sample C2. The dotted lines in figures show the cubic CdTe reflections with relative intensities.

QDs (sample C0) with an average size of $3.6 \mathrm{~nm}$, CdTe/CdS core/shell QDs (sample C1) with an average size of $4.2 \mathrm{~nm}$, and CdTe/CdS core/shell QDs (sample C2) with an average size of $4.7 \mathrm{~nm}$. Figure 5 approximately match the theoretical thickness calculated from the amount of injected stock solution, which gives strong evidence for the epitaxial growth of CdS onto the CdTe cores and excludes the independent nucleation of CdS. It is quite evident that these QDs are close to spherical particles with excellent monodispersity.

3.2. PL Lifetime of CdTe/CdS QDs. Photoluminescence lifetime measurement is another useful approach to confirm a change in the surface state of QDs. Figure 6 shows the timeresolved luminescence decay curves of TGA-capped CdTe cores and CdTe/CdS QDs (as-prepared), and Figure 7 shows the time-resolved luminescence decay curves of TGA-capped CdTe cores and CdTe/CdS QDs (130 days): (a) luminescence decay curves of samples A0, A1, and A2; (b) luminescence decay curves of samples B0, B1, and B2; (c) luminescence decay curves of samples $\mathrm{C} 0, \mathrm{C} 1$, and $\mathrm{C} 2$. For all samples, time-resolved emission measurements have been done at 


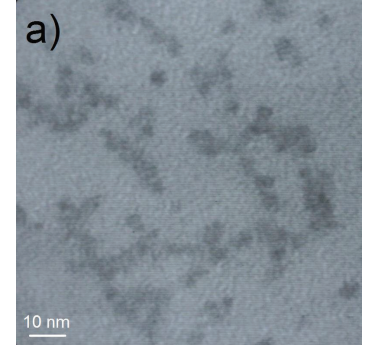

(a)

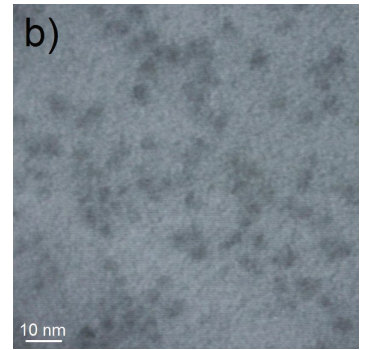

(b)

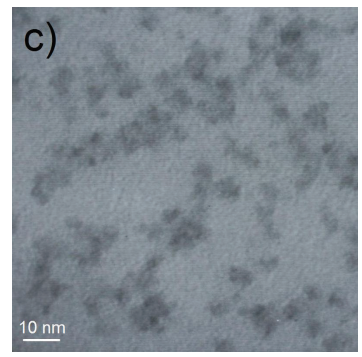

(c)

Figure 5: TEM images of TGA-capped CdTe, CdTe/CdS core/shell QDs: (a) sample C0 $(3.6 \mathrm{~nm})$; (b) sample C1 $(4.2 \mathrm{~nm})$; and (c) sample C2 $(4.7 \mathrm{~nm})$.

their peak wavelengths in steady state PL spectra. The decay curves can be well fitted to a biexponential model described by the equation as follows:

$$
F(t)=\mathrm{A}+\mathrm{B}_{1} \exp \left(-\frac{t}{\tau_{1}}\right)+\mathrm{B}_{2} \exp \left(-\frac{t}{\tau_{2}}\right)
$$

where $\tau_{1}$ and $\tau_{2}\left(\tau_{1}<\tau_{2}\right)$ represent the time constants, and $\mathrm{B}_{1}$ and $\mathrm{B}_{2}$ represent the amplitudes of the fast and slow components, respectively. Average lifetime $\tau$ was calculated using the equation below [21]:

$$
\tau=\frac{\left(\mathrm{B}_{1} \tau_{1}^{2}+\mathrm{B}_{2} \tau_{2}^{2}\right)}{\left(\mathrm{B}_{1} \tau_{1}+\mathrm{B}_{2} \tau_{2}\right)}
$$

The fitted values of the parameters $\mathrm{B}_{1}, \mathrm{~B}_{2}, \tau_{1}, \tau_{2}$, and $\tau$ are summarized in Table 2 (as-prepared) and Table 3 (130 days).

In Figures 6 and 7, Tables 2 and 3, a CdS shell coating makes CdTe/CdS QDs a long lifetime compared to the CdTe cores due to drastic increase in the charge carrier in the core/shell structure. With increasing the shell thickness, the average lifetime of the core/shell QDs was also prolonged. The spatial separation of electron and hole can result in a decrease of the wave function overlap and thus a longer lifetime of the charge recombination is expected in type II heterostructures. For epitaxial growth of the shell over the core, it is very important to have less lattice mismatch between core and shell material.

In Table 2, the lifetime of sample B0 has a significant increase from as-prepared to 130 days, this phenomenon is ascribed to the increased surface defects, in which the defects led the lifetime to be prolonged. To the contrary, the lifetimes of samples $\mathrm{A} 0$ and $\mathrm{C} 0$ have a slight decrease, from

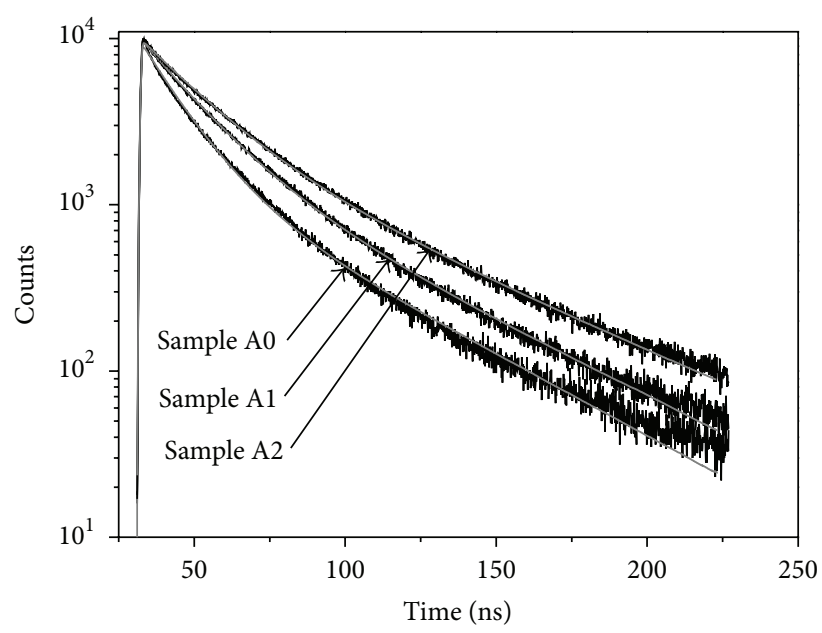

(a)

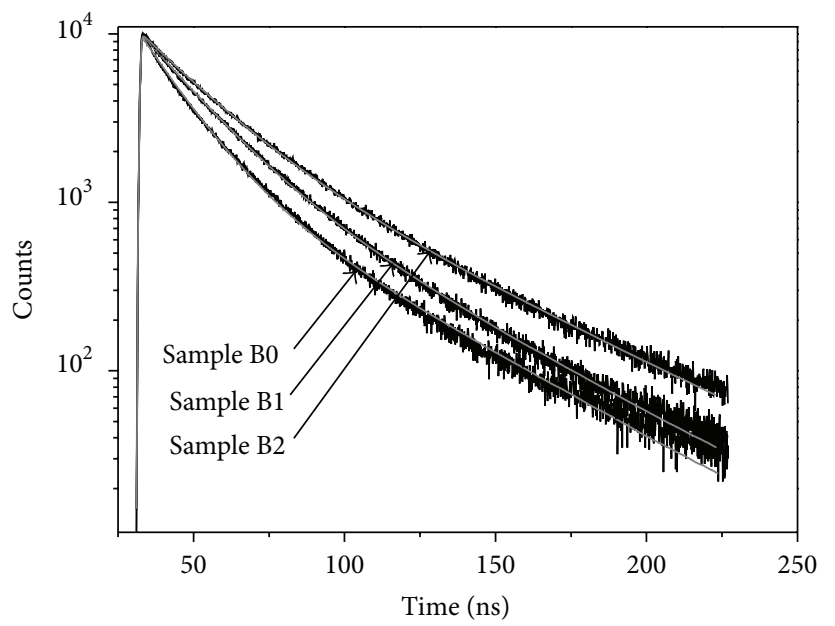

(b)

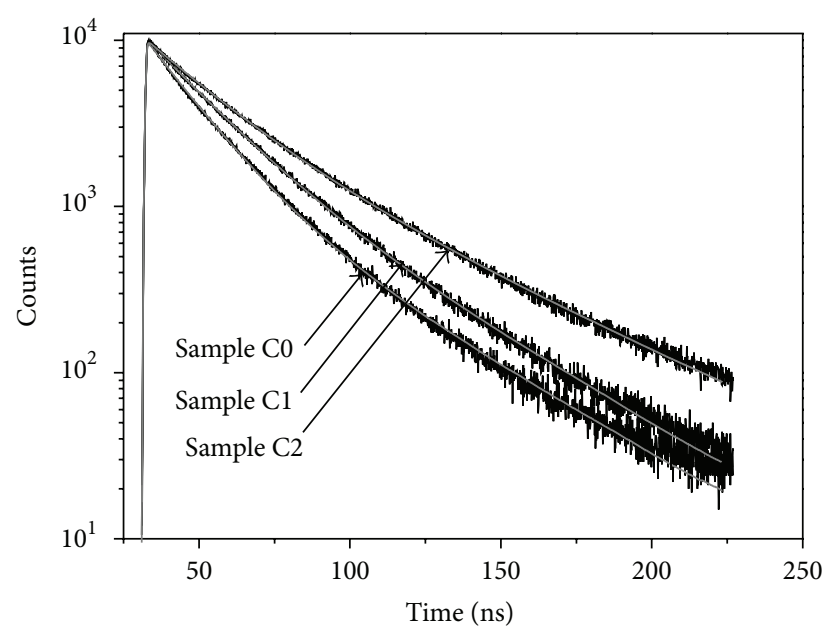

(c)

FiguRE 6: Time-resolved luminescence decay curves (measured at maximum emission peak, $\lambda_{\mathrm{ex}}=370 \mathrm{~nm}$ ) of TGA-capped CdTe, and CdTe/CdS core/shell QDs (as-prepared). (a) samples A0, A1, and A2; (b) samples B0, B1, and B2; (c) samples C0, C1, and C2. Reproduced curves for data shown in Table 2 are plotted as thin gray lines. 


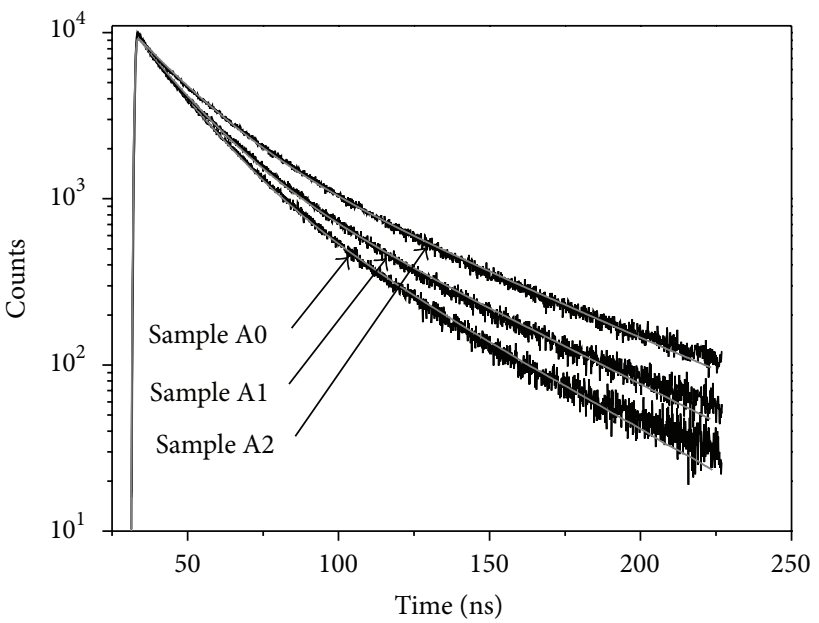

(a)

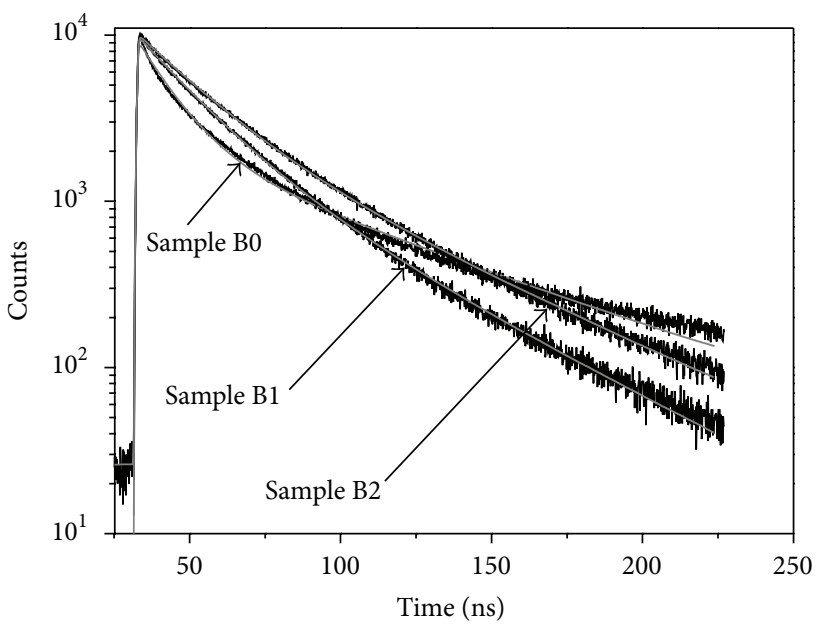

(b)

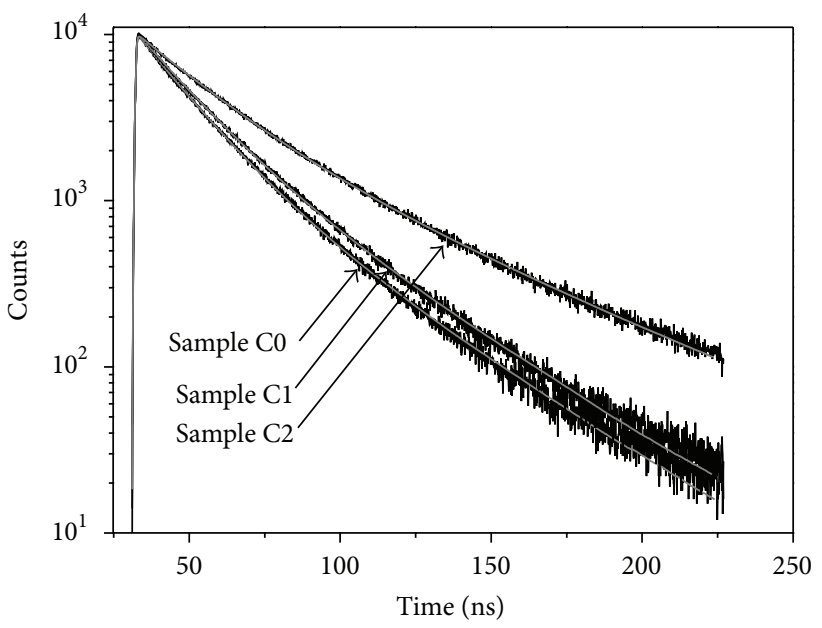

(c)

Figure 7: Time-resolved luminescence decay curves (measured at maximum emission peak, $\lambda_{\mathrm{ex}}=370 \mathrm{~nm}$ ) of TGA-capped CdTe, and $\mathrm{CdTe} / \mathrm{CdS}$ core/shell QDs (130 days). (a) samples A0, A1, and A2; (b) samples B0, B1, and B2; (c) samples C0, C1, and C2. Reproduced curves for data shown in Table 3 are plotted as thin gray lines.
TABLE 2: Time constants $\tau_{1}$ and $\tau_{2}$, components $\mathrm{B}_{1}$ and $\mathrm{B}_{2}$, and average lifetime $\tau$ of as-prepared TGA-capped CdTe, and CdTe/CdS core/shell QDs (samples shown in Table 1 (as-prepared)).

\begin{tabular}{lccccc}
\hline Sample & $\mathrm{B}_{1}$ & $\tau_{1} / \mathrm{ns}$ & $\mathrm{B}_{2}$ & $\tau_{2} / \mathrm{ns}$ & $\tau / \mathrm{ns}$ \\
\hline A0 & 56.73 & 12.99 & 43.27 & 43.26 & 34.7 \\
A1 & 56.21 & 17.37 & 43.79 & 47.58 & 37.9 \\
A2 & 50.33 & 20.09 & 49.67 & 54.77 & 45.4 \\
B0 & 59.65 & 14.26 & 40.35 & 42.80 & 33.4 \\
B1 & 61.38 & 18.54 & 38.62 & 45.35 & 34.8 \\
B2 & 52.90 & 21.01 & 47.10 & 51.06 & 41.6 \\
C0 & 66.91 & 16.44 & 33.09 & 41.09 & 30.1 \\
C1 & 63.15 & 20.24 & 36.85 & 42.14 & 32.3 \\
C2 & 51.92 & 23.36 & 48.08 & 52.87 & 43.3 \\
\hline
\end{tabular}

TABLE 3: Time constants $\tau_{1}$ and $\tau_{2}$, components $\mathrm{B}_{1}$ and $\mathrm{B}_{2}$, and average lifetime $\tau$ of TGA-capped $\mathrm{CdTe}$, and $\mathrm{CdTe} / \mathrm{CdS}$ core/shell QDs (samples shown in Table 1, stored at room temperature for 130 days).

\begin{tabular}{lccccc}
\hline Sample & $\mathrm{B}_{1}$ & $\tau_{1} / \mathrm{ns}$ & $\mathrm{B}_{2}$ & $\tau_{2} / \mathrm{ns}$ & $\tau / \mathrm{ns}$ \\
\hline $\mathrm{A} 0$ & 57.12 & 15.32 & 42.88 & 40.53 & 32.1 \\
$\mathrm{~A} 1$ & 49.93 & 15.86 & 50.07 & 47.01 & 39.2 \\
$\mathrm{~A} 2$ & 44.89 & 18.46 & 55.11 & 55.05 & 47.2 \\
B0 & 38.52 & 11.91 & 61.48 & 62.93 & 57.5 \\
B1 & 55.48 & 18.60 & 44.52 & 45.24 & 36.2 \\
B2 & 51.12 & 21.30 & 48.88 & 53.58 & 44.1 \\
C0 & 62.62 & 17.01 & 37.38 & 37.16 & 28.4 \\
C1 & 55.99 & 17.97 & 44.01 & 37.68 & 30.2 \\
C2 & 51.42 & 24.13 & 48.58 & 57.39 & 47.2 \\
\hline
\end{tabular}

$34.7,31.0,30.1 \mathrm{~ns}$ ( 0 day) to $32.1,28.4,28.4$ (130 days); this phenomenon is due to the decreased surface defects caused by light etching. The lifetime of most $\mathrm{CdTe} / \mathrm{CdS}$ core/shell QDs (sample A1, A2, B1, B2, and C2) increased from asprepared to 130 days, which is corresponding with the red shift of $\lambda_{\mathrm{em}}$ from as-prepared to 130 days. With increasing the time of storagetime, the average lifetime of the core/shell QDs was also prolonged. For Ostwald ripening mechanism, the sizes of $\mathrm{CdTe} / \mathrm{CdS}$ core/shell QDs increased, which give rise to the increase of the charge separation in the CdTe/CdS core/shell QDs. It is evident that the average PL decay times increase steadily with increasing size of the QDs, a tendency that is generally observed for II-VI QDs [22].

\section{Conclusion}

TGA-capped CdTe/CdS core/shell QDs in an aqueous solution were synthesized by depositing a CdS shell on CdTe cores in an aqueous solution. The epitaxial growth of the CdS shell was confirmed by the red-shifted absorption and emission spectra of the CdTe/CdS core/shell QDs and the XRD patterns of the QDs. The CdTe cores and CdTe/CdS core/shell QDs exhibited tunable PL peak wavelengths from 541 to $631 \mathrm{~nm}$ for as-prepared synthesized samples (as-prepared) and from 556 to $633 \mathrm{~nm}$ for a storage time of 130 days, respectively. The red-shifted degree of the CdTe cores is larger 
than that of the CdTe/CdS core/shell QDs. It proved that $\mathrm{CdTe} / \mathrm{CdS}$ core/shell QDs have better PL properties than $\mathrm{CdTe}$ cores. Systematic investigations of PL lifetime demonstrated that the CdTe/CdS core/shell QDs revealed a prolonged average PL lifetime and PL stability compared with CdTe cores. The research on the PL stability of stored CdTe and CdTe/CdS core/shell QDs has a significant meaning for the application of QDs.

\section{Acknowledgments}

This work was supported in part by the Program for Taishan Scholars, Project from National Science Foundation of China (21071061), Project from Natural Science Foundation of Shandong Province (ZR2010EZ001), Outstanding Young Scientists Foundation Grant of Shandong Province (BS2010CL004), and Graduate Student Innovation Foundation of University of Jinan (YCX10018).

\section{References}

[1] D. R. Larson, W. R. Zipfel, R. M. Williams et al., "Watersoluble quantum dots for multiphoton fluorescence imaging in vivo," Science, vol. 300, no. 5624, pp. 1434-1436, 2003.

[2] A. P. Alivisatos, "Semiconductor clusters, nanocrystals, and quantum dots," Science, vol. 271, no. 5251, pp. 933-937, 1996.

[3] J. Liu, H. Li, W. Wang et al., "Use of ester-terminated polyamidoamine dendrimers for stabilizing quantum dots in aqueous solutions," Small, vol. 2, no. 8-9, pp. 999-1002, 2006.

[4] Q. Lu, S. S. Hu, D. W. Pang, and Z. K. He, "Direct electrochemistry and electrocatalysis with hemoglobin in water-soluble quantum dots film on glassy carbon electrode ," Chemical Communications, vol. 20, no. 20, pp. 2584-2585, 2005.

[5] Y. X. Xu, J. G. Liang, C. G. Hu, F. S. Wang, S. Hu, and Z. K. He, "A hydrogen peroxide biosensor based on the direct electrochemistry of hemoglobin modified with quantum dots," Journal of Biological Inorganic Chemistry, vol. 12, no. 3, pp. 421-427, 2007.

[6] C. B. Murray, D. J. Norris, and M. G. Bawendi, "Synthesis and characterization of nearly monodisperse CdE (E = S, Se, $\mathrm{Te}$ ) semiconductor nanocrystallites," Journal of the American Chemical Society, vol. 115, no. 19, pp. 8706-8715, 1993.

[7] S. Wang, N. Mamedova, N. A. Kotov, W. Chen, and J. Studer, "Antigen/Antibody Immunocomplex from CdTe Nanoparticle Bioconjugates," Nano Letters, vol. 2, no. 8, pp. 817-822, 2002.

[8] A. L. Rogach, L. Katsikas, A. Kornowski, D. Su, A. Eychmuller, and H. Weller, "Synthesis and characterization of thiolstabilized CdTe nanocrystals," Berichte der Bunsengesellschaft für Physikalische Chemie, vol. 100, pp. 1772-1778, 1996.

[9] Y. Zhang, J. He, P. N. Wang et al., "Time-dependent photoluminescence blue shift of the quantum dots in living cells: effect of oxidation by singlet oxygen," Journal of the American Chemical Society, vol. 128, no. 41, pp. 13396-13401, 2006.

[10] M. A. Hines and P. Guyot-Sionnest, "Synthesis and characterization of strongly luminescing ZnS-capped CdSe nanocrystals," Journal of Physical Chemistry, vol. 100, no. 2, pp. 468471, 1996.

[11] B. O. Dabbousi, J. Rodriguez-Viejo, F. V. Mikulec et al., "(CdSe)ZnS core-shell quantum dots: synthesis and characterization of a size series of highly luminescent nanocrystallites," Journal of Physical Chemistry B, vol. 101, no. 46, pp. 9463 9475, 1997.
[12] M. Danek, K. F. Jensen, C. B. Murray, and M. G. Bawendi, "Synthesis of luminescent thin-film CdSe/ZnSe quantum dot composites using CdSe quantum dots passivated with an overlayer of ZnSe," Chemistry of Materials, vol. 8, no. 1, pp. 173-180, 1996.

[13] P. Reiss, J. Bleuse, and A. Pron, "Highly Luminescent CdSe/ZnSe core/shell nanocrystals of low size dispersion," Nano Letters, vol. 2, no. 7, pp. 781-784, 2002.

[14] X. Peng, M. C. Schlamp, A. V. Kadavanich, and A. P. Alivisatos, "Epitaxial growth of highly luminescent CdSe/CdS core/shell nanocrystals with photostability and electronic accessibility," Journal of the American Chemical Society, vol. 119, no. 30, pp. 7019-7029, 1997.

[15] T. Trindade, P. O'Brien, and N. L. Pickett, "Nanocrystalline semiconductors: synthesis, properties, and perspectives," Chemistry of Materials, vol. 13, no. 11, pp. 3843-3858, 2001.

[16] Y. Yu, Y. Lai, X. Zheng, J. Wu, Z. Long, and C. Liang, "Synthesis of functionalized CdTe/CdS QDs for spectrofluorimetric detection of BSA," Spectrochimica Acta Part A, vol. 68, no. 5, pp. 1356-1361, 2007.

[17] C. L. Wang, H. Zhang, J. H. Zhang, M. J. Li, H. Z. Sun, and B. Yang, "Application of ultrasonic irradiation in aqueous synthesis of highly fluorescent CdTe/CdS core-shell nanocrystals," The Journal of Physical Chemistry C, vol. 111, no. 6, pp. 2465-2469, 2007.

[18] H. Peng, L. Zhang, C. Soeller, and J. Travas-Sejdic, "Preparation of water-soluble CdTe/CdS core/shell quantum dots with enhanced photostability," Journal of Luminescence, vol. 127, no. 2, pp. 721-726, 2007.

[19] Z. M. Yuan, A. Y. Zhang, Y. Q. Cao, J. Yang, Y. N. Zhu, and P. Yang, "Effect of mercaptocarboxylic acids on luminescent properties of CdTe quantum dots," Journal of Fluorescence, vol. 22, no. 1, pp. 121-127, 2011.

[20] H. Bao, Y. Gong, Z. Li, and M. Gao, "Enhancement effect of illumination on the photoluminescence of water-soluble CdTe nanocrystals: toward highly fluorescent $\mathrm{CdTe} / \mathrm{CdS}$ core-shell structure," Chemistry of Materials, vol. 16, no. 20, pp. 38533859, 2004.

[21] M. Jones, J. Nedeljkovic, R. J. Ellingson, A. J. Nozik, and G. Rumbles, "Photoenhancement of luminescence in colloidal CdSe quantum dot solutions," Journal of Physical Chemistry B, vol. 107, no. 41, pp. 11346-11352, 2003.

[22] S. Rawalekar, S. Kaniyankandy, S. Verma, and H. N. Ghosh, "Ultrafast charge carrier relaxation and charge transfer dynamics of $\mathrm{CdTe} / \mathrm{CdS}$ core-shell quantum dots as studied by femtosecond transient absorption spectroscopy," Journal of Physical Chemistry C, vol. 114, no. 3, pp. 1460-1466, 2010. 


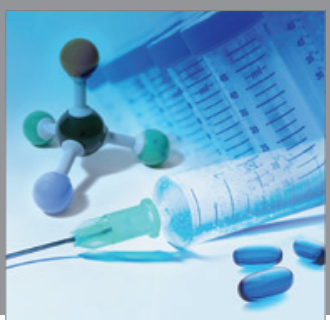

International Journal of

Medicinal Chemistry

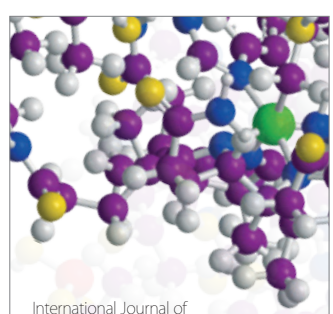

Carbohydrate Chemistry

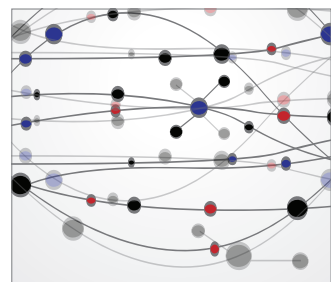

The Scientific World Journal
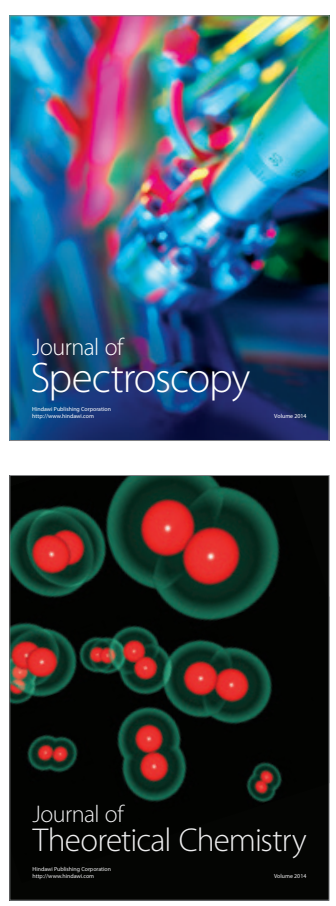
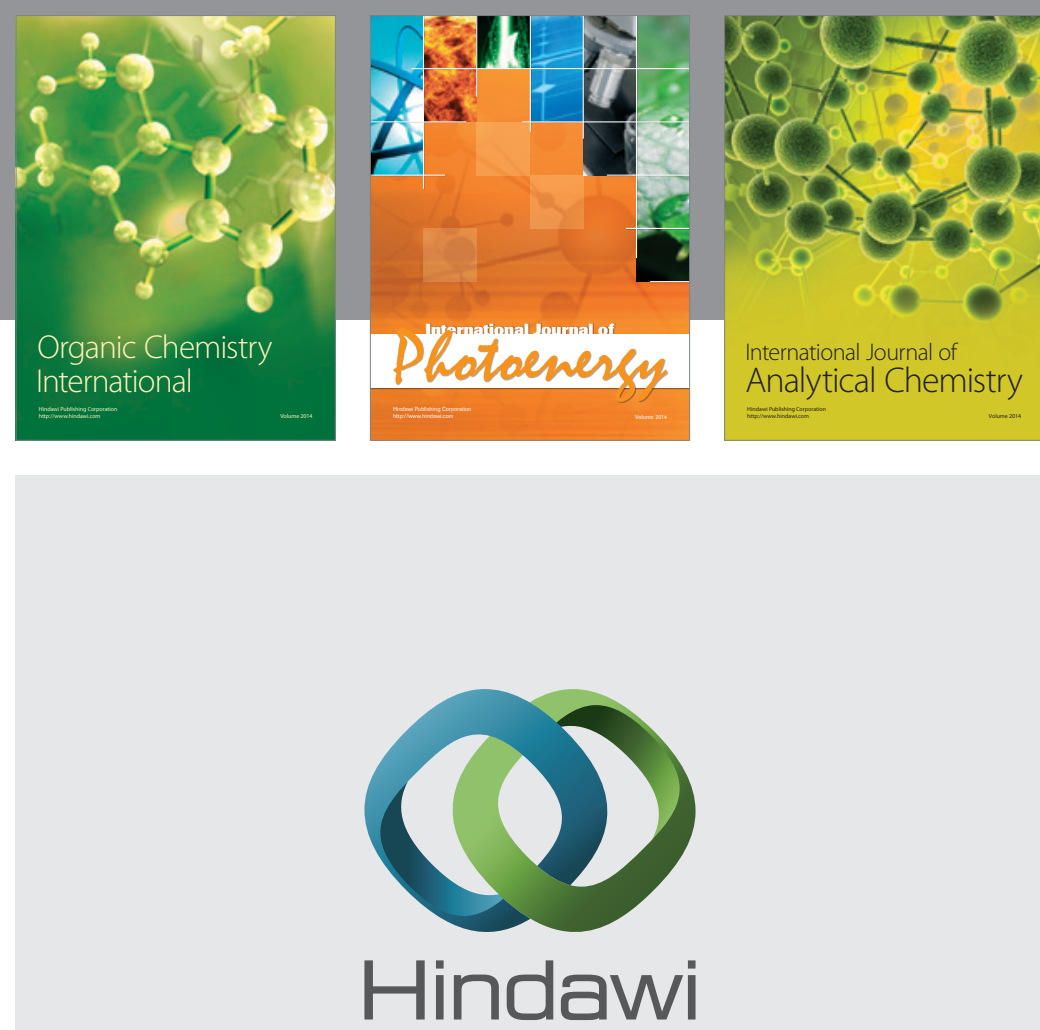

Submit your manuscripts at

http://www.hindawi.com
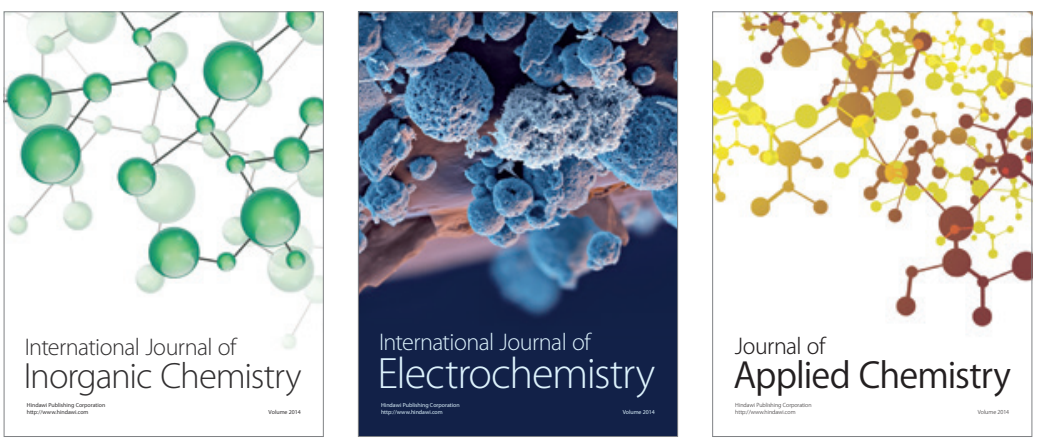

Journal of

Applied Chemistry
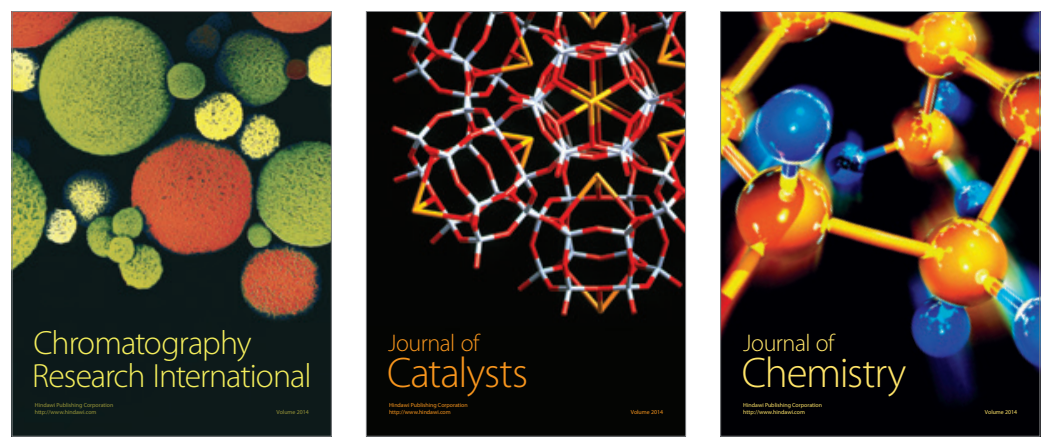
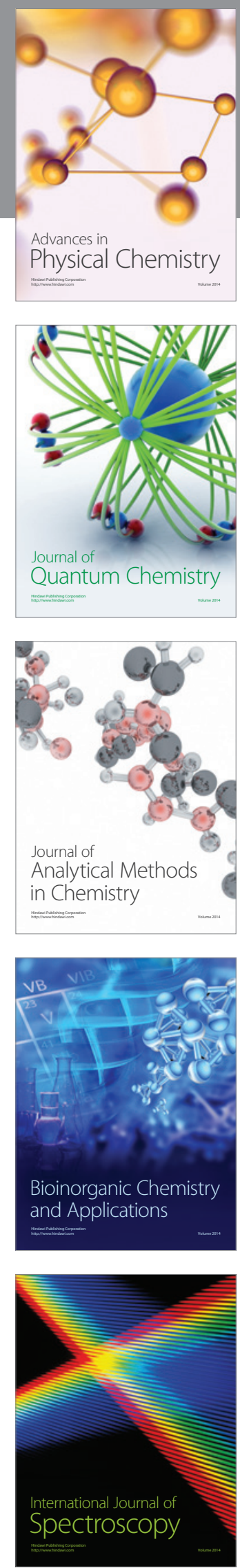